\title{
Correction
}

\section{Mixed Student Ideas about Mechanisms of Human Weight Loss}

The authors of "Mixed Student Ideas about Mechanisms of Human Weight Loss" (CBE-Life Sciences Education, 18(3), ar37, DOI:10.1187/cbe.18-11-0227) have made a correction to a sentence in the Methods.

The original text (found on the top of page 3, second line from the top of the second column):

We collected a total of 2445 student responses from students in introductory biology classes for life science majors, because these courses often cover energy and matter transformations in cellular respiration.

Correction

We collected a total of 3100 student responses from students in introductory biology classes for life science majors, because these courses often cover energy and matter transformations in cellular respiration.

The HTML and PDF versions were corrected on the CBE-Life Sciences Education website on November 4, 2020. These corrections may not appear on copies of the article that reside on other websites. 